\title{
Use of Animation to Stimulate Students' Interests in Mechanism Design
}

\author{
Daniel M. Chen
}

Central Michigan University

\section{Introduction}

Mechanism design is one of the most important applications in an integrated computer-aided engineering (CAE) software. Few products designed today do not have moving parts. Mechanism design in CAE allows the designer to put the solid parts of a machine into motion without actually building one. In addition, the parameters such as interference, limits of motion, and geometric properties that include displacement, velocity, and acceleration can be easily obtained. With such a powerful tool, students can enhance their problem solving skills and understand the concepts of mechanism design better along the way.

The course of Advanced Computer-Aided Design offered at Central Michigan University employs such a design tool called SDRC/I-DEAS. Although this software is sophisticated in terms of capabilities, it is not difficult to learn due to its visual feedback at every step. This is crucial for students in engineering technology who learn best when they can see things and work with them. The capability of animation provided by the software stimulates great interests from students. This article discusses how the animation is used to help students understand some of the more challenging concepts in mechanism design. The focuses of this investigation are on: (1) how the problems are designed to stimulate students' interests with the use of animation, and (2) how the learning process is enhanced in mechanism design as a result.

\section{Animation of A Mechanism in I-DEAS}

This section discusses how the animation of a mechanism (or mechanisms) is achieved in IDEAS. The following represents the procedure students must follow in order to solve and animate the mechanism [1,3]. The solid-based parts are first created and used to define the assemblies and sub-assemblies. The assembly hierarchy is displayed in a form that lets students build the assembly according to the relationship among parts [2]. The title of the mechanism serves as the parent for the hierarchy tree. The part names are added to this tree to define the assembly. Sub-assemblies, which consist of groups of parts, can also be added to the tree if necessary. Then, students apply joints/constraints and grounds to the system and define motion input to one or more joints.

The above is the preparation required before the internal solver of I-DEAS can be used to solve the mechanism. In the internal solver window, students need only to enter the end time and number of steps in that same period of time. The results generated by the internal solver include 
geometric properties and animation. Students usually animate the motion first after a mechanism is solved. The capability of 3D animation allows configurations to be automatically stored for the specific steps of motion. To complete an animation sequence, each moving part in the system will either translate or rotate (or both) until the number of configurations is completed. In terms of displaying geometric properties, the graphs of linear and angular displacement, velocity, and acceleration of any moving part can be plotted as a variable of time. With the software, students can also easily check the limit of motion and interference.

\section{Design of Problems}

In general, the problems used in the course of Advanced Computer-Aided Design include the following four different categories:

A. To identify the motion of a machine with some of its major joints/constraints specified.

B. To find all the required joints/constraints for a machine with its motion defined.

C. To identify the replacement of a mechanism that would generate the same motion.

D. To find the input motion required to create a specific output motion.

Figure 1 depicts an example in category A. A welding robot is modeled with the following specifications: a rotational body to provide the circular motion and two hydraulic cylinders to provide the planar motion. A revolute joint is applied between the body and the base that is grounded, and a translational joint between the piston and the cylinder for each of the two hydraulic cylinders. Each joint is represented by a coordinate system in green. Students were required to create all additional joints/constraints to allow the welding gun to move as designed. Figure 1 shows there are six more revolute and translational joints required. Three input motions include a constant angular velocity for the revolute joint, and a constant linear velocity for each of the translational joint.

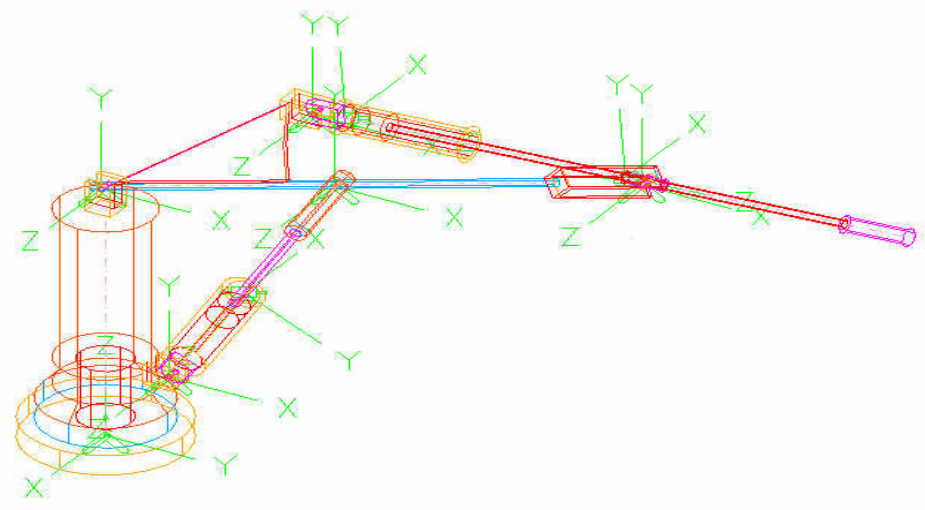

Figure 1. Welding Robot

Figure 2 depicts an example in category B. An automobile is moving along a racing track. Students need to identify all the required joints/constraints, so the automobile can generate the motion as defined. As the automobile moves forward, it needs to translate and rotate because two of its wheels on one side must be always in contact with the edge of the track. A cam/follower constraint is ideal for this application. Each wheel, which is represented by a knife-edge follower, is forced to slide against the edge of the track that is basically a grounded 
cam profile. A revolute joint is then applied between the pin (attached to a block) and the automobile to allow the automobile to rotate. The input motion, which is an angular velocity assigned to the revolute joint between the arm and the grounded cylinder, drives the automobile forward. As the automobile moves along the track, it also needs to move toward and away from the cylinder. This is achieved by implementing a translational joint between the pin and the arm. In order to allow the animation to look more realistic, the cylinder, the arm, and the pin could be made invisible via the CAD function of translucency.

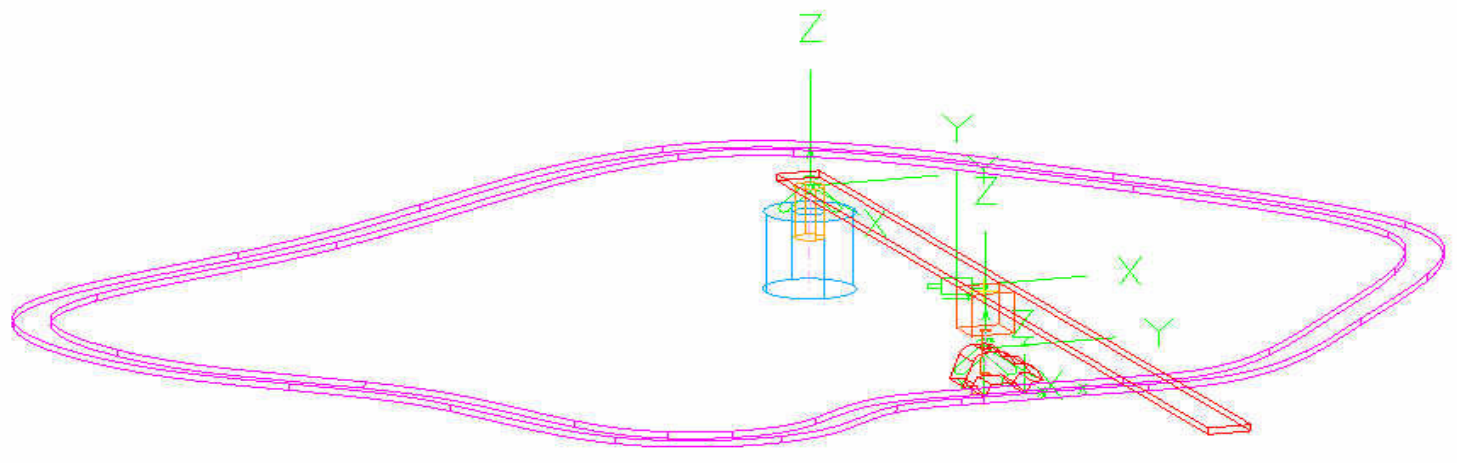

Figure 2. Automobile and Race Track

Figure 3 shows an example in category C. A toy robot is to be modeled, so each of its two forelegs lifts when moving forward. A link with a slot in Figure 3a is suggested for the foreleg, so the link would not only slide against a fixed pin but also rotate about it. Students were required to find a substitute mechanism to achieve the same motion. The design of forelegs for the toy robot in Figure $3 b$ was the most popular replacement students came up with. Each foreleg consists of a cylinder and a piston with single-end rod. The cylinder rotates while the piston slides.

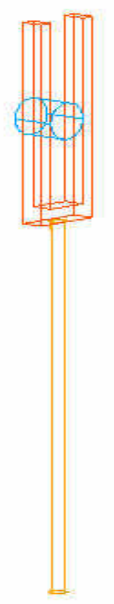

(a)

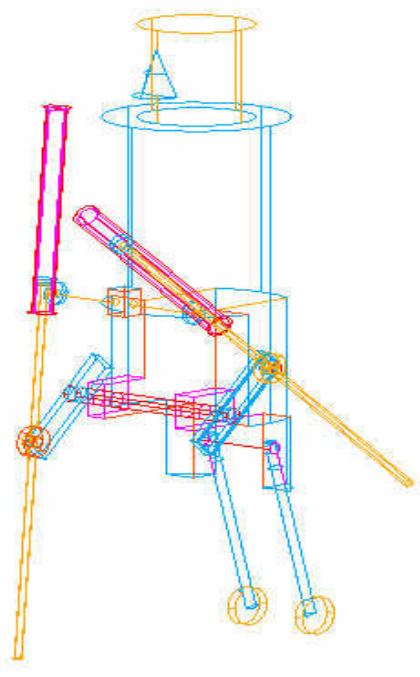

(b)

Figure 3. Toy Robot

Proceedings of the 2002 American Society for Engineering Education Annual Conference \& Exposition 
Figure 4 shows an example in category D. A rocket is to be launched from the ground. It requires an acceleration as the input motion for the translational joint between the rocket and the ground (grounded cylinder). No matter what the input motion is, a constant or a time-dependent, the geometric properties can be easily obtained as soon as the mechanism is solved. Figure 5 depicts the obtained geometric properties that include curves of displacement, velocity, and acceleration. This problem can be made more interesting if the rocket is also to spin unevenly. The challenge would be with the input motion required for the additional revolute joint. Students need to learn how to deal with the input angular velocity that is a function of time.

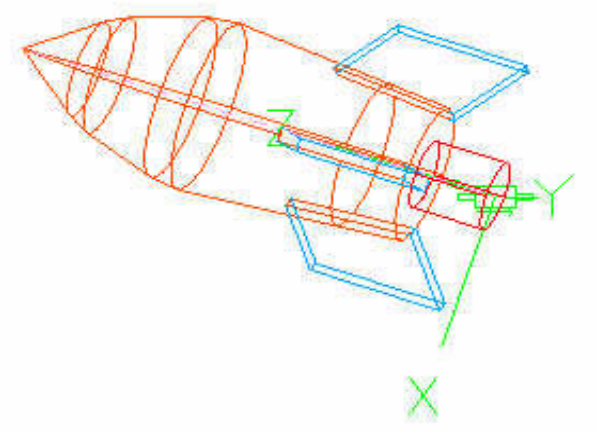

Figure 4. Rocket with a Translational Joint

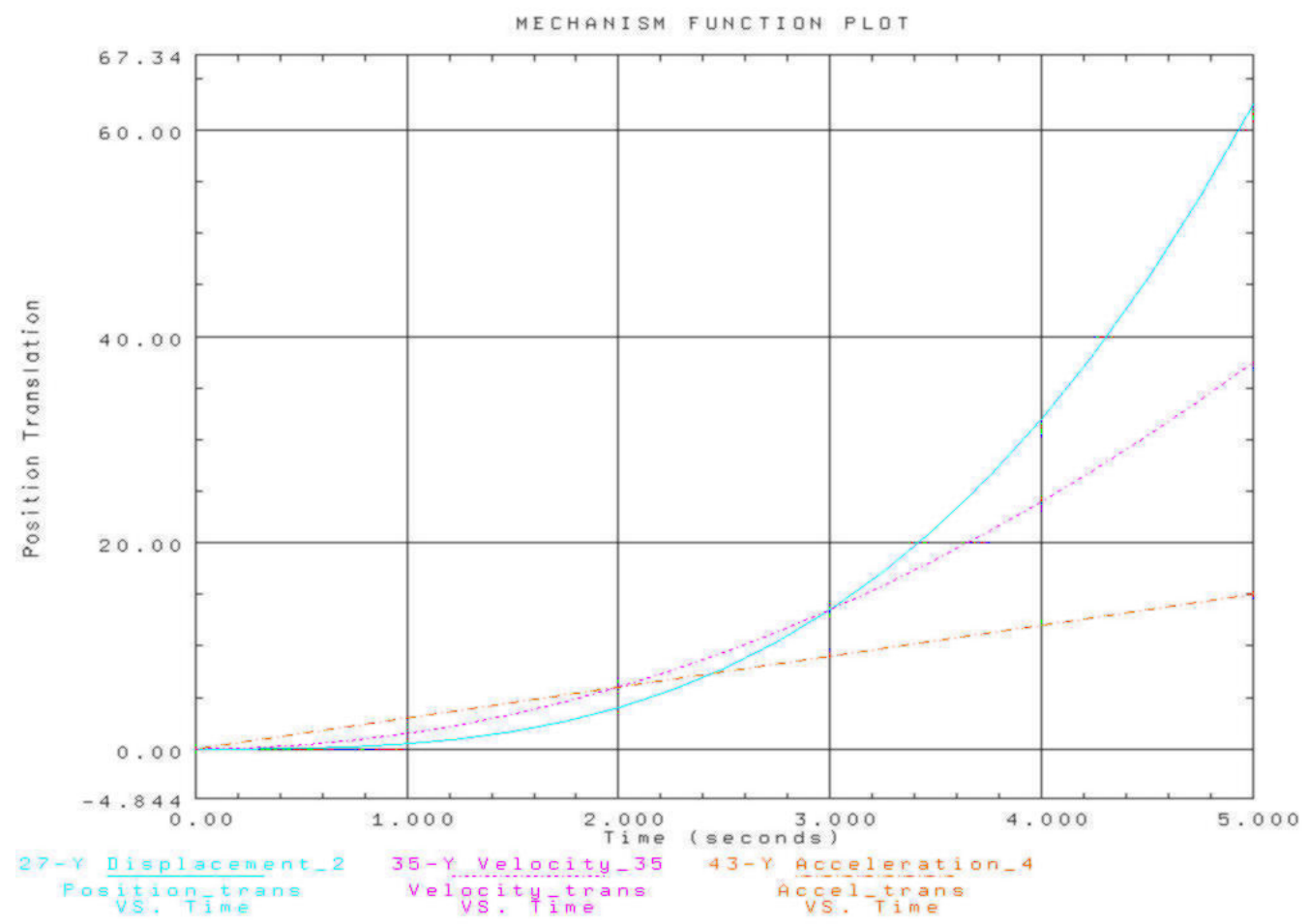

Figure 5. Curves of Displacement, Velocity, and Acceleration 


\section{Discussion}

The following table summarizes what students would have learned by completing the assigned problems that are similar to the examples above.

\begin{tabular}{|l|l|}
\hline Welding Robot & $\begin{array}{l}\text { How to complete the design of a machine with limited } \\
\text { information provided }\end{array}$ \\
\hline $\begin{array}{l}\text { Automobile/Race } \\
\text { Track }\end{array}$ & $\begin{array}{l}\text { How to design a machine from scratch to generate a specific } \\
\text { motion }\end{array}$ \\
\hline Toy Robot & $\begin{array}{l}\text { How to design a mechanism for substitution that generates } \\
\text { the same motion }\end{array}$ \\
\hline Rocket & $\begin{array}{l}\text { How to deal with the required input motion, constant or } \\
\text { time-dependent }\end{array}$ \\
\hline
\end{tabular}

No matter what problem in any category was assigned, students were very excited as soon as they viewed their design animated. This excitement was the major driving force for students to keep up their efforts.

It's easy to see that the design of problems in most categories is not difficult. Their design relies on the ideas from the mechanisms used in well-known machines. According to the instructor's observation, although students found the problems in all categories interesting, the problems in category B seemed to attract more interests. This type of problems focuses on one major point how to use the joints/constraints provided by the software to create a motion that can be easily generated using an animated video clip. The design of category B problems is more timeconsuming. Because, the instructor needs to make sure that the problem as designed can be animated using input motion and joints/constraints provided by the software before it's given to students.

\section{Conclusion}

The students' learning process can be greatly enhanced in mechanism design by solving problems similar to those previously discussed. This is primarily due to the software's capabilities in animation and quick display of results. Once the mechanism is solved, students can use the animation to check the motion of each part relative to the others in the mechanism. They can have the instant access of the graphs of displacement, velocity, and acceleration for all the parts, which are difficult to obtain with the traditional 2D drafting approach. With the software, students can use the tremendous amount of time saved for further design revisions, and therefore, have a better understanding of the physical problem.

Bibliography

1. Mark H. Lawry, The I-DEAS Student Guide, Structural Dynamics Research Corporation, 2000.

2. The Fundamentals of I-DEAS Course Guide, Structural Dynamics Research Corporation, 1996. 
3. The I-DEAS Course Guide - Mechanism Design Course, Structural Dynamics Research Corporation, 1997.

\section{DANIEL CHEN}

Daniel M. Chen is Professor of Industrial and Engineering Technology at Central Michigan University. He has taught various courses in Mechanical Engineering Technology during the last sixteen years. Currently, near half of his teaching load is in Computer-Aided Design/Computer-Aided Engineering. Dr. Chen is a registered Professional Engineer in Michigan. He received his Ph.D. in Mechanical Engineering from Kansas State University in 1984. He received his B.S. and M.S. in that same discipline from Taipei Institute of Technology and South Dakota School of Mines and Technology, respectively. 